Tania Gaspar ${ }^{1}$

Ana Cerqueira $^{2}$

Catia Branquinho ${ }^{3}$

Margarida Gaspar de Matos ${ }^{4}$
${ }^{1}$ Professor com Agregação - (Diretora do Instituto de Psicologia e Ciências da Educação/ CLISSIS Universidade Lusíada de Lisboa ISAMB/ Universidade de Lisboa) - AdHoc - AdHoc.

${ }^{2}$ Mestre em Psicologia Clínica - (Investigadora Aventura Social/Universidade de Lisboa; CLISSIS/Universidade Lusíada de Lisboa).

${ }^{3}$ Mestre em Psicologia Clínica - (Investigadora e Doutoranda FMH/Aventura Social/Universidade de Lisboa).

${ }^{4}$ Professora Catedrática com Agregação FMH/ Aventura Social/Universidade de Lisboa ISAMB/ Universidade de Lisboa - (FMH/Aventura Social/Universidade de Lisboa ISAMB/ Universidade de Lisboa).

Correspondência:

Tania Gaspar

Instituição: Universidade Lusíada de Lisboa, Rua da Junqueira, n188-198, 1349-001 Lisboa, Portugal

CEP: 1349-001

E-mail:tania.gaspar.barra@gmail.com Este artigo foi submetido no SGP (Sistema de Gestão de Publicações) da RBTC em 12 de Janeiro de 2018. cod. 601

Artigo aceito em 25 de Julho de 2019.

\section{Programa competências integradas: perceção dos professores e psicólogos}

\section{Integrated Skills Program: Perception Of Teachers And Psychologists}

\section{RESUMO}

Pretende-se estudar o impacto da implementação de um programa de promoção de competências sociais e emocionais num centro de educação e desenvolvimento, tendo em conta a perceção dos professores e psicólogos. Este estudo inclui a perceção de professores e de psicólogos sobre 755 alunos com idades entre os 9 e os 21 anos, $24,0 \%$ com 12 ou menos anos, $26,0 \%$ dos 13 aos 15 anos e $50,1 \%$ com 16 ou mais anos de idade. $59,5 \%$ são meninos e $9,3 \%$ encontram-se em regime de programa educativo individual (PEI). $\mathrm{O}$ estudo da escala de competências socioemocionais revela boas propriedades psicométricas e revela ser um bom contributo para a avaliação de competências na infância e adolescência. Segundo a perceção dos professores e psicólogos que implementaram o programa, verifica-se o aumento de competências na maioria das dimensões da escala, nomeadamente, ao nível das competências globais, nas competências avançadas, nas competências em lidar com sentimentos, competências alternativas à agressividade e competências de planeamento entre o momento pré e pós-intervenção. $O$ estudo contribui para a investigação e avaliação de intervenção em crianças e adolescentes, especialmente na prevenção e promoção de competências pessoais e sociais e desenvolvimento saudável.

Palavras-chave: Promoção de saúde; Competências pessoais e sociais; Avaliação de programas.

\section{ABSTRACT}

The aim is to study the impact of the implementation of a program to promote social and emotional competences in an education center, based on teachers and psychologist perception. This study included teachers' perceptions of 755 students aged 9 to 21 years, $24.0 \%$ with 12 or less years, $26.0 \%$ from 13 to 15 years and $50.1 \%$ with 16 or more years of schooling. age. $59.5 \%$ are boys and $9.3 \%$ are under the Individual Educational Program (PEI). The study of the socio-emotional skills scale reveals good psychometric properties and reveals to be a good contribution to the evaluation of competences in childhood and adolescence. According to the perception of the teachers and psychologists who implemented the program, there is an increase in competences in most of the dimensions of the scale, namely global competences, advanced skills, skills in dealing with feelings, skills that are alternative to aggression and planning between the pre and post intervention moments. The study contributes to the research and evaluation of intervention in children and adolescents, especially in the prevention and promotion of personal and social skills and healthy development.

Keywords: Health promotion; Social and personal skills; Programs assessment. 


\section{INTRODUÇÃO}

No processo de desenvolvimento integral da criança, identificam-se dois contextos fundamentais, a escola e a família. Constituindo-se a escola como o contexto privilegiado para o desenvolvimento de competências e estratégias de adaptação às várias situações e desafios vivenciados no quotidiano, ao nível da relação com as dificuldades de regulação do comportamento pessoal e social, adaptação à escola ou insucesso escolar (Choi, 2018; David, Paixão, \& da Silva, 2015; Gaspar, Cerqueira, Branquinho, \& Matos, 2018a; Goldman, Stamler Kleinman, Kerner, \& Lewis, 2016; Matos \& Sampaio, 2009).

Crianças e adolescentes com maior nível de competências pessoais e sociais tendem a desenvolver estratégias de gestão do risco e estratégias de coping mais eficazes e estão também associadas a uma melhor perceção de bem-estar e saúde mental (Gaspar, Matos, Ribeiro, Leal, Erhart, \& Ravens-Sieberer, 2012; Gaspar, Bilimória, Albergaria, \& Matos, 2016; Gaspar, Rebelo, Mendonça, Albergaria, \& Matos, 2014; Gaspar, Matos, PaisRibeiro, Leal, \& Albergaria, 2014; Matos, Simões, Gaspar, \& equipa Aventura Social, 2012; McConnel, Memetovic, \& Richardson, 2014).

A aprendizagem das competências socioemocionais inicia-se quando o nascimento e prolonga-se ao longo da vida, através da imitação social dos modelos sociais com os quais o sujeito se relaciona ao longo da sua vida (Bandura, 1976). As relações interpessoais saudáveis e estáveis são um dos elementos fundamentais que promove o desenvolvimento positivo das crianças e adolescentes (Goldman et al., 2016).

A escola apresenta-se como um contexto fulcral na preparação para a vida devendo, para além da abordagem educacional, a par da família e do meio envolvente, proporcionar um desenvolvimento emocional e social mais saudável (Almeida, Valentini, \& Berleze, 2009; Gaspar, 2010; Goldman et al. 2016).

Os programas de promoção de competências pessoais e sociais são considerados importantes formas de promover a saúde e qualidade de vida na escola, através do desenvolvimento das competências do jovem para lidar consigo e com os outros, situações de stress e desafios proporcionados pelo ambiente (Durlak, Weissberg, Dymnicki, Taylor, \& Schellinger, 2011; Gaspar, Matos, Ribeiro, \& Leal, 2006).

Adaptados às necessidades dos jovens, ao seu desenvolvimento e cultura, estes programas podem assumir um papel curativo ou remediativo, no âmbito da minimização de problemas individuais, uma direção preventiva com foco no trabalho de evitamento de determinado problema ou desenvolvimental, na promoção da utilização de competências pessoais e sociais ajustadas a uma determinada situação ou contexto (Matos \& Tomé, 2012). A promoção destas competências permite uma adaptação mais ajustada aos diversos contextos e 0 estabelecimento de relacionamentos interpessoais mais positivos e adequados (Domitrovich, Durlak, Staley, \& Weissber, 2017; Weissberg, Durlak, Domitrovich, \& Gullotta, 2015).
As necessidades e diferenças individuais, nomeadamente, ao nível da idade, género, necessidades educativas especiais, envolvimento e sucesso escolar entre outras têm influência nas competências socioemocionais dos jovens e devem ser integradas e ser consideradas no planeamento da intervenção (Bauminger \& Kimhi-Kind, 2008; Bauminger, Edelsztein, \& Morash, 2005; Garner, Mahatmya, Brown, \& Vesely, 2014; Hampel \& Petermann, 2005; 2006; Hen \& Goroshit, 2014; Kavale \& Forness, 1996; Kavale \& Mostert, 2004; Mammarella et al., 2014; Nowicki, Brown, \& Stepien, 2014; Sahoo, Biswas, \& Padhy, 2015; Vaughn, Elbaum, \& Boardman, 2001).

Nos programas de intervenção de promoção de competências pessoais e sociais, considera-se elementar o treino das diferentes competências: comunicação e relacionamento interpessoal; expor e defender as suas ideias/opiniões e direitos de forma assertiva; negociação; gestão de conflitos; resolução de problemas; tomada de decisões e expetativas face ao futuro (Matos, 2008; Matos et al., 2009). Existem evidências na literatura que apontam para a escola como ocupando um lugar de destaque no que diz respeito às intervenções preventivas que visam o aumento de competências para lidar com os desafios diários, sendo um local passível de influenciar o desenvolvimento psicossocial dos jovens (Goldman et al., 2016; Ungar, Russel, \& Connelly, 2014). Alguns destes desafios incluem o insucesso académico, relacionamentos interpessoais (Chase, Hilliard, Geldhof, Warren, \& Lerner, 2014; Moksnes, Espnes, \& Haugan, 2014), comportamentos ligados à saúde (tais como adições, comportamentos alimentares e sexuais) (Campbell \& Peebles, 2014; Morris \& Rushwan, 2015; Spear, 2015) e ao nível da gestão emocional (Riediger \& Klipker, 2014). Intervenções de promoção de competências sociais e emocionais com base na escola, para além de desenvolver estratégias para lidar com problemas, dão uma visão mais positiva da saúde e aumentam o bem-estar dos mais jovens (Sancassiani et al., 2015).

Os programas em contexto escolar têm sido alvo de avaliações no sentido de atestar a sua eficácia na promoção de competências pessoais, ao nível da comunicação, resolução de problemas, regulação emocional, relacionamentos interpessoais e expectativas futuras e gestão/organização do horário de trabalho e de lazer. Através de dados provenientes de uma metaanálise de programas extra-escolares, que visam a promoção de competências pessoais e sociais de crianças e adolescente, foi possível verificar que os participantes obtiveram um aumento significativo ao nível da autoperceção e vínculo escolar, comportamentos sociais positivos, desempenho escolar e nível de realização académica e, também, uma redução significativa ao nível dos comportamentos problemáticos, comparativamente com o grupo de controlo (Blum \& Dick, 2013; Matos et al., 2012; Matos, Tomé, Gaspar, Cicognani, \& Moreno, 2016; Reddy, 2013).

Fatores como o ser menina (Eastabrook, Flynn, \& Hollenstein, 2014;Gaspar et al.,2012), ter necessidades educativas especiais (Gaspar, Bilimória, Albergaria, \& Matos, 2016), o insucesso escolar (Gaspar, Rebelo, Mendonça, 
Albergaria, \& Matos, 2014) ou um estatuto socioeconómico baixo (Gaspar \& Balancho, 2016; Gaspar, Matos, Luszczynska, \& De Wit, 2016; Reddy, 2013), podem ser considerados fatores de risco, no que diz respeito à saúde e bem-estar subjetivo.

O impacto destes fatores de risco ou as suas consequências ao nível desenvolvimental e psicológico pode ser minimizado através da promoção de competências pessoais e sociais, que permitam um melhor enfrentamento das situações e um aumento das oportunidades para um desenvolvimento positivo (Blum \& Dick, 2013; Gaspar et al., 2018a; Taylor, Oberle, Durlak, \& Weissberg, 2017; WHO, 2014). Outra questão a ter em conta prende-se com o facto da idade se refletir na forma como os jovens percecionam as suas competências e, por consequência, a sua capacidade de fazer autoavaliações mais realistas (Coelho, Sousa, Marchante, \& Romão, 2015; Tracey \& Ward, 1998). Ao mesmo tempo, a adolescência é um período marcado por diversas alterações e desafios que requerem uma capacidade adaptativa por parte dos jovens (Booker \& Dunsmore, 2016; Compas et al., 2017; Cracco, Goossens, \& Braet, 2017; Modecki, Zimmer-Gembeck, \& Guerra, 2017; Zimmermann \& Iwanski, 2014).

O principal objetivo é estudar o impacto da implementação de um programa de promoção de competências sociais e emocionais num centro de educação e desenvolvimento, tendo como base a perceção dos professores e psicólogos.

\section{MÉTODO}

\section{Participantes}

Neste estudo participaram 60 professores e psicólogos que reportaram a sua avaliação das competências socioemocionais de 755 alunos com idades entre os 9 e os 21 anos, $24,0 \%$ com 12 ou menos anos, $26,0 \%$ dos 13 aos 15 anos e $50,1 \%$ com 16 ou mais anos de idade. $59,5 \%$ meninos $(n=449)$ e $40,5 \%$ meninas $(n=306)$. Todos são estudantes de um Centro Educativo e de Desenvolvimento situado em Lisboa, Portugal e são alvo de um programa de competências integradas (CSI). Do total dos estudantes envolvidos, 9,3\% $(n=70)$ encontram-se em regime de Programa Educativo Individual (PEI) para crianças com necessidades educativas especiais.

\section{INSTRUMENTO}

O instrumento utilizado foi a adaptação da check-list - aprendizagem estruturada em skills sociais de Goldstein e McGinnis (1997) traduzida e adaptada do Inglês para Português por Matos (2005) resultando numa escala de medida de competências sociais e pessoais. $\mathrm{O}$ instrumento obtido deve ser preenchido pelos profissionais (professores, educadores e psicólogos) acerca dos estudantes.

Este instrumento avalia as competências sociais em seis dimensões distintas, nomeadamente: Competências Sociais Básicas (ouvir, ter uma conversa, dizer obrigado, etc.); Competências Sociais Avançadas (pedir ajuda, pedir desculpa, etc.); Competências para Lidar com Sentimentos (conhecer os sentimentos, expressar os sentimentos, etc.); Competências Alternativas à Agressividade (pedir permissão, usar autocontrolo, etc.); Competências para Lidar com o Stress (fazer uma queixa, responder a uma queixa, etc.); e Competências de Planeamento (tomar uma decisão, definir objetivos, etc.). Cada uma destas dimensões é constituída por um conjunto de itens. Cada item tem como opção de resposta uma escala de Likert composta por 5 níveis diferentes: Nunca (1), Quase Nunca (2), Algumas Vezes (3), Muitas Vezes (4), Sempre (5). Na Tabela 1 são apresentadas e desenvolvidas as dimensões da escala de competências socioemocionais. Apresenta boas propriedades psicométricas que variam entre alpha de Cronbach de 0,80 na dimensão "Competências avançadas (CA)" e 0,95 na dimensão "Competências planeamento (CP)." O alpha de Cronbach da escala total é de 0, 97 (Tabela 2).

Tabela 1. Dimensões da escala de competências socioemocionais e respetivas competências

\begin{tabular}{|c|c|}
\hline Dimensões & Competências \\
\hline $\begin{array}{l}\text { Competências } \\
\text { básicas }\end{array}$ & $\begin{array}{l}\text { Ouvir; Iniciar uma conversa; Ter uma conversa; } \\
\text { Fazer perguntas; Dizer obrigado; Apresentar- } \\
\text { se; Apresentar outras pessoas; Dar um elogio }\end{array}$ \\
\hline $\begin{array}{l}\text { Competências } \\
\text { sociais avançadas }\end{array}$ & $\begin{array}{c}\text { Pedir ajuda; Dar instruções; Seguir instruções; } \\
\text { Pedir desculpa }\end{array}$ \\
\hline $\begin{array}{l}\text { Competências } \\
\text { para lidar com } \\
\text { sentimentos }\end{array}$ & $\begin{array}{l}\text { Conhecer os sentimentos; Expressar os } \\
\text { sentimentos; Compreender os sentimentos } \\
\text { dos outros; Expressão de sentimentos } \\
\text { adequados à situação }\end{array}$ \\
\hline $\begin{array}{l}\text { Competências } \\
\text { alternativas à } \\
\text { agressividade }\end{array}$ & $\begin{array}{l}\text { Pedir permissão; Partilhar alguma coisa; } \\
\text { Ajudar os outros; Pedir ajuda; Negociar; } \\
\text { Combinar; Usar autocontrolo; Defender os } \\
\text { seus direitos }\end{array}$ \\
\hline $\begin{array}{l}\text { Competências para } \\
\text { lidar com o stress }\end{array}$ & $\begin{array}{l}\text { Fazer uma queixa; Responder a uma queixa; } \\
\text { Lidar com a exclusão; Responder à persuasão; } \\
\text { Lidar com uma acusação; Lidar com a pressão } \\
\text { do grupo; Lidar com os clientes/utentes; } \\
\text { Lidar com os colegas de trabalho; Lidar com o } \\
\text { patrão; Lidar com as rotinas do quotidiano }\end{array}$ \\
\hline $\begin{array}{l}\text { Competências de } \\
\text { planeamento }\end{array}$ & $\begin{array}{c}\text { Saber o que tem de fazer numa determinada } \\
\text { situação; Identificar o que está a causar o } \\
\text { problema; Definir objetivos; Reconhecer as } \\
\text { suas capacidades; Reunir informação sobre } \\
\text { os problemas; Organizar os problemas pela } \\
\text { importância; Tomar uma decisão; Concentrar- } \\
\text { se numa tarefa }\end{array}$ \\
\hline
\end{tabular}

Tabela 2. Descritivas - Distribuição nas dimensões do instrumento Escala de competências socioemocionais (ECSE)

\begin{tabular}{lccc}
\hline & M & DP & A \\
\hline Competências básicas (CB) & 3.42 & 0.74 & 0.92 \\
Competências avançadas (CA) & 3.25 & 0.72 & 0.80 \\
$\begin{array}{l}\text { Competências lidar com senti- } \\
\text { mentos (CLS) }\end{array}$ & 3.24 & 0.72 & 0.89 \\
$\begin{array}{l}\text { Competências alternativas agres- } \\
\text { sividade (CAA) }\end{array}$ & 3.41 & 0.65 & 0.83 \\
Competências lidar stress (CLS) & 3.12 & 0.61 & 0.89 \\
Competências planeamento (CP) & 3.15 & 0.74 & 0.95 \\
Competências totais (CT) & 3.25 & 0.56 & 0.97 \\
\hline
\end{tabular}




\section{Procedimento}

A presente investigação-ação teve lugar num Centro de Educação e Desenvolvimento que desenvolveu junto de todos os seus estudantes um programa de promoção de competências pessoais e sociais denominado CSI - Programa de Competências Sociais Integradas. Este programa surgiu em 2011 com o objetivo de reunir todas as áreas trabalhadas em diversos projetos e outros temas pertinentes para a populaçãoalvo, aplicando-se a todos os educandos com mais de 4 anos. O programa é desenvolvido por dois aplicadores por cada grupo de educandos, um elemento da equipa educativa (professor/educador) e um elemento da equipa técnica com formação na área da psicologia. O grupo de aplicadores é supervisionado pelo grupo de referência CSI, com a função de acompanhamento, formação e avaliação do programa.

O programa CSI tem um período anual, um número médio de 22 sessões e conta com dois temas centrais: educação sexual e desenvolvimento vocacional, desenvolvendo-se os restantes temas nas áreas da alimentação, educação para as novas tecnologias, prevenção das substâncias psicoativas, prevenção de comportamentos violentos, cidadania, etc., dependentemente das características do grupo, utilizando-se manuais e materiais como histórias, músicas, jogos e dinâmicas para as sessões no âmbito dos temas centrais e também nas sessões referentes à prevenção de substâncias psicoativas e nos restantes temas um conjunto de orientações que apoiam na seleção dos materiais a utilizar.

O objetivo geral do CSI é desenvolver competências pessoais e sociais relacionadas ao autoconhecimento, à comunicação, às relações interpessoais e à tomada de decisões. Essas competências são desenvolvidas através de dinâmicas relacionais, a experiência e exploração de comunicação, dificuldades e conflitos ligados a relacionamentos interpessoais e comportamentos de saúde, relacionados à sexualidade, abuso de substâncias, estilos de vida e saúde mental. Os principais conteúdos abordados com os participantes são: Comunicação interpessoal; Assertividade; Gestão de conflitos; Resolução de problemas, e Gestão emocional.

As atividades desenvolvidas dependeram das características e necessidades específicas de cada grupo. Foram utilizados manuais e diferentes materiais incluindo histórias, música, jogos e dinâmicas de grupo.

Cada sessão apresentou a mesma estrutura global, incluindo uma atividade inicial (eventos relevantes desde a última sessão) e quebra de gelo, uma atividade relacionada ao conteúdo e objetivo da sessão, uma atividade de cooperação e uma atividade final de reflexão da sessão e trabalho para desenvolver até a próxima sessão.

Para uma avaliação do programa CSI, a escala de competências socioemocionais (ECSE) foi aplicada aos professores ou psicólogos dinamizadores do Programa CSI para obter a sua perceção das competências dos estudantes. Numa fase anterior ao início do desenvolvimento do programa (pré- teste) e após o término (pós-teste), por forma a avaliar o impacto do programa nos grupos ao nível dosskills: sociais básicos, sociais avançados, para lidar com sentimentos, alternativos à agressividade, lidar com o stress e de planeamento.

O estudo cumpriu os procedimentos éticos necessários e exigidos para o estudo em questão sendo parte integrante do plano de atividades estratégicas para o Centro Educativo, nomeadamente, a autorização à Presidente da Direção do Centro Educativo, de seguida, a autorização aos pais para o envolvimento dos seus educandos na investigação-ação, aceitação de participação por parte de professores e psicólogos e os estudantes foram informados dos objetivos do estudo, do seu caracter voluntário e confidencial.

\section{RESULTADOS}

No estudo realizado no âmbito do impacto do desenvolvimento do Programa CSI, um total de 775 alunos foram avaliados por 60 aplicadores (professores e psicólogos). Pretendeu-se estudar o impacto do desenvolvimento do programa de promoção de competências pessoais e sociais através da aplicação da escala de competências socioemocionais (ECSE) na análise pré e pós-teste, nos estudantes de um Centro de Educação e Desenvolvimento.

Como forma de compreender a qualidade do instrumento utilizado, foi ainda realizada a análise das propriedades do instrumento utilizado, a escala de competências socioemocionais (ECSE).

O alpha de Cronbach revelou uma fiabilidade muito boa na escala total ( $\alpha=0.97$ ), assim como na subescala competências básicas $(a=0.92)$ e competências de planeamento ( $\alpha=0.95$ ), uma boa fiabilidade na subescala sentimentos $(a=0.89)$, alternativas à agressividade $(a=0.83)$, lidar com o stress $(\alpha=0.89)$ e na subescala competências avançadas $(a=0.80)$.

Segundo a perceção dos profissionais os estudantes apresentam valores positivos ao nível das suas competências socioemocionais. Sendo as competências básicas e as competências alternativas à agressividade as mais valoradas positivamente pelos profissionais (Tabela 2).

As dimensões da escala apresentam correlações estatisticamente significativas, salientando-se as correlações mais elevadas $(r>0.80)$ entre as competências básicas e as competências avançadas, e as diferentes dimensões e a escala total (com exceção da correlação com a dimensão de competências para lidar com stress que é inferior a 0.80) (Tabela 3).

Foram encontradas diferenças estatisticamente significativas ao nível das competências entre os meninos e as meninas. Segundo os profissionais, as meninas, apresentam mais competências socioemocionais do que os meninos em todas as dimensões da escala (Tabela 4).

Foram encontradas algumas diferenças estatisticamente significativas ao nível das competências entre os estudantes mais novos e mais velhos. Segundo os profissionais, os estudantes 
Tabela 3. Correlações entre competências básicas, avançadas, lidar com sentimentos, alternativas à agressividade, lidar com o stress, planeamento e totais

\begin{tabular}{|c|c|c|c|c|c|c|}
\hline Variáveis & $\mathrm{CB}$ & CA & CLS & CAA & CLS & $\mathrm{CP}$ \\
\hline Comp. básicas & -- & & & & & \\
\hline Comp. avançadas & $0.81^{* * *}$ & -- & & & & \\
\hline Comp. alternativas agressividade & $0.73^{* * *}$ & $0.78^{* * *}$ & $0.73^{* * * *}$ & -- & & \\
\hline Comp. lidar com stress & $0.37^{* * *}$ & $0.40^{* * *}$ & $0.40^{* * *}$ & $0.45^{* * *}$ & -- & \\
\hline Comp. totais & $0.86^{* * *}$ & $0.87^{* * *}$ & $0.81 * * *$ & $0.87^{* * *}$ & $0.66^{* * *}$ & $0.84^{* * *}$ \\
\hline
\end{tabular}

$56^{* \star *} p<0,001$

Tabela 4. ANOVA - Competências básicas, avançadas, lidar com sentimentos, alternativas à agressividade, lidar com o stress, planeamento e totais e diferenças de género

\begin{tabular}{|c|c|c|c|c|c|}
\hline \multirow[t]{2}{*}{ Dimensões } & \multicolumn{2}{|c|}{ Menino } & \multicolumn{2}{|c|}{ Menina } & \multirow{2}{*}{$\mathbf{F}$} \\
\hline & $\mathbf{M}$ & DP & $\mathbf{M}$ & DP & \\
\hline Competências básicas & 3.32 & 0.73 & 3.56 & 0.72 & $19.85^{* * *}$ \\
\hline Competências avançadas & 3.16 & 0.72 & 3.39 & 0.69 & $18.19^{* * *}$ \\
\hline $\begin{array}{l}\text { Competências lidar com } \\
\text { sentimentos }\end{array}$ & 3.16 & 0.69 & 3.36 & 0.74 & $13.66^{* * *}$ \\
\hline $\begin{array}{l}\text { Competências alternativas } \\
\text { a agressividade }\end{array}$ & 3.31 & 0.63 & 3.56 & 0.65 & $27.09^{* * *}$ \\
\hline Competências lidar stress & 3.08 & 0.57 & 3.17 & 0.66 & $4.03^{*}$ \\
\hline $\begin{array}{l}\text { Competências } \\
\text { planeamento }\end{array}$ & 3.08 & 0.73 & 3.25 & 0.75 & $9.48^{* *}$ \\
\hline Competências totais & 3.18 & 0.54 & 3.37 & 0.56 & $21.02^{* * *}$ \\
\hline
\end{tabular}

${ }^{* * *} p<0,001 ;{ }^{* *} p<0,01 ;{ }^{*} p<0,05$

mais novos, apresentam mais competências socioemocionais ao nível das competências avançadas e nas competências relacionadas com gestão dos sentimentos do que os estudantes mais velhos (13 anos ou mais). Nas restantes dimensões da escala não foram identificadas diferenças estatisticamente significativas nos diferentes grupos de idade (Tabela 5).
Foram encontradas diferenças estatisticamente significativas ao nível das competências entre os estudantes com e sem necessidades educativas especiais. Segundo os professores/psicólogos, os alunos com PEl, apresentam menos competências socioemocionais do que os estudantes sem PEI em todas as dimensões da escala, com exceção à dimensão relacionada com a gestão de stress que não foram encontradas diferenças estatisticamente significativas (Tabela 6).

Tabela 6. ANOVA - Competências básicas, avançadas, lidar com sentimentos, alternativas à agressividade, lidar com o stress, planeamento e totais e diferenças PEI

\begin{tabular}{lccccc}
\hline \multirow{2}{*}{ Dimensões } & \multicolumn{2}{c}{ Sim } & \multicolumn{2}{c}{ Não } & \multirow{2}{*}{ F } \\
\cline { 2 - 5 } & M & DP & M & DP & \\
\hline Competências básicas & 3.04 & 0.70 & 3.46 & 0.73 & $20.53^{* * *}$ \\
Competências avançadas & 2.93 & 0.67 & 3.29 & 0.72 & $15.54^{* * *}$ \\
$\begin{array}{l}\text { Competências lidar com } \\
\text { sentimentos }\end{array}$ & 2.93 & 0.73 & 3.27 & 0.71 & $14.78^{* * *}$ \\
$\begin{array}{l}\text { Competências alternativas } \\
\text { agressividade }\end{array}$ & 3.15 & 0.66 & 3.44 & 0.64 & $13.11^{* * *}$ \\
$\begin{array}{l}\text { Competências lidar stress } \\
\text { Competências }\end{array}$ & 3.02 & 0.54 & 3.13 & 0.62 & 1.95 \\
$\begin{array}{l}\text { planeamento } \\
\text { Competências totais }\end{array}$ & 2.49 & 0.58 & 3.21 & 0.73 & $63.99^{* * *}$ \\
\cline { 1 - 4 } & 2.92 & 0.53 & 3.29 & 0.55 & $28.31^{* * *}$ \\
\hline
\end{tabular}

${ }^{* * *} p<0,001$

Tabela 5. ANOVA - Competências básicas, avançadas, lidar com sentimentos, alternativas à agressividade, lidar com o stress, planeamento e totais e diferenças de idade

\begin{tabular}{|c|c|c|c|c|c|c|c|}
\hline \multirow{2}{*}{ Dimensões } & \multicolumn{2}{|c|}{ Até 12 anos } & \multicolumn{2}{|c|}{ 13-15 anos } & \multicolumn{2}{|c|}{16 ou mais anos } & \multirow{2}{*}{$\mathbf{F}$} \\
\hline & $\mathbf{M}$ & DP & M & DP & M & DP & \\
\hline Competências básicas & 3.47 & 0.74 & 3.39 & 0.74 & 3.41 & 0.74 & 0.74 \\
\hline Competências lidar com sentimentos & 3.43 & 0.74 & 3.15 & 0.76 & 3.20 & 0.67 & $8.81^{* * *}$ \\
\hline Competências alternativas agressividade & 3.45 & 0.63 & 3.45 & 0.70 & 3.37 & 0.63 & 1.24 \\
\hline Competências planeamento & 3.13 & 0.70 & 3.06 & 0.72 & 3.20 & 0.77 & 2.32 \\
\hline Competências totais & 3.30 & 0.54 & 3.22 & 0.56 & 3.26 & 0.56 & 0.91 \\
\hline
\end{tabular}

${ }^{* * *} p<0,001 ;{ }^{* *} p<0,01$ 
Segundo os profissionais, os estudantes alteraram o seu nível de competências socioemocionais após o programa de promoção de competências. Verifica-se o aumento de competências na maioria das dimensões da escala, nomeadamente ao nível das competências globais, nas competências avançadas, nas competências em lidar com sentimentos, competências alternativas à agressividade e competências de planeamento entre o momento pré e pós-intervenção. Em relação às competências básicas e competências em lidar com o stress não se verificaram alterações estatisticamente significativas nos dois momentos de avaliação (Tabela 7).

Tabela 7. ANOVA - Competências básicas, avançadas, lidar com sentimentos, alternativas à agressividade, lidar com o stress, planeamento e totais e diferenças pré e pós-teste

\begin{tabular}{|c|c|c|c|c|c|}
\hline \multirow{2}{*}{ Dimensões } & \multicolumn{2}{|c|}{ Pré-teste } & \multicolumn{2}{|c|}{ Pós-teste } & \multirow{2}{*}{$\mathbf{F}$} \\
\hline & M & DP & M & DP & \\
\hline Competências básicas & 3.42 & 0.74 & 3.50 & 0.76 & 3.79 \\
\hline Competências avançadas & 3.25 & 0.72 & 3.37 & 0.73 & $8.67^{* *}$ \\
\hline $\begin{array}{l}\text { Competências lidar com } \\
\text { sentimentos }\end{array}$ & 3.24 & 0.72 & 3.33 & 0.76 & $4.69^{*}$ \\
\hline $\begin{array}{l}\text { Competências alternativas } \\
\text { agressividade }\end{array}$ & 3.41 & 0.65 & 3.53 & 0.66 & $10.40^{* *}$ \\
\hline Competências lidar stress & 3.11 & 0.61 & 3.18 & 0.66 & 3.67 \\
\hline $\begin{array}{l}\text { Competências } \\
\text { planeamento }\end{array}$ & 3.15 & 0.74 & 3.27 & 0.79 & $9.35^{* *}$ \\
\hline Competências totais & 3.26 & 0.56 & 3.35 & 0.60 & $8.05^{* *}$ \\
\hline
\end{tabular}

${ }^{\star *} p<0,01 ;{ }^{*} p<0,05$

\section{DISCUSSÃO}

O presente estudo tem como principal objetivo estudar o impacto da implementação de um programa de promoção de competências sociais e emocionais num centro de educação e desenvolvimento, tendo em conta a perceção de professores e psicólogos.

Em relação à Escala de Competências Socioemocionais que foi utilizada para avaliar as competências integradas, verifica-se que inclui seis dimensões das competências socioemocionais, nomeadamente, Competências Sociais Básicas, Competências Sociais Avançadas, Competências para Lidar com Sentimentos, Competências Alternativas à Agressividade, Competências para Lidar com o Stress e Competências de Planeamento, e apresenta boas qualidades psicométricas. As dimensões encontradas são semelhantes às encontradas noutros estudos e instrumentos de avaliação de competências socioemocionais em crianças e adolescentes (Gaspar \& Matos, 2015; Matos, 2005).

A escala permite o estudo das competências socioemocionais dos estudantes. Através da análise dos resultados verifica-se que os estudantes apresentam valores positivos em todas as dimensões da escala, especialmente ao nível das competências básicas e das competências alternativas à agressividade.

Segundo os profissionais, as meninas, apresentam mais competências socioemocionais do que os meninos em todas as dimensões da escala.Segundo os profissionais, os estudantes mais novos, apresentam mais competências socioemocionais ao nível das competências avançadas e nas competências relacionadas com gestão dos sentimentos do que os estudantes mais velhos (13 anos ou mais). Estes resultados podem ser compreendidos numa perspetiva desenvolvimental, as crianças mais novas ainda possuem pouca experiência para realizar julgamentos autónomos relativos às suas capacidades, o que resulta em valores de avaliações mais altos.

As crianças mais novas baseiam a sua avaliação no feedback social que recebem, enquanto as crianças mais velhas têm em conta as suas próprias expectativas e padrões internalizados de sucesso e fracasso, para além do feedback social recebido. A competência percebida é formada através da combinação de quatro constructos: experiências passadas, dificuldade ou desafio associado ao resultado, interações e reforço transmitido através das relações interpessoais e motivação intrínseca (Harter, 1978). Assim, nas crianças mais velhas, o sentimento de competência tende a ser mais autodeterminado e mais realista. À medida que a criança se vai desenvolvendo verifica-se uma estabilização ao nível da competência percebida o que resulta numa avaliação de competência mais ajustada à realidade. Assim, as crianças mais velhas passam a ter em conta diversos fatores que influenciam o seu desempenho, como o feedback das pessoas significativas, as experiências passadas e a forma positiva ou negativa como as vivenciaram, os tipos de tarefas e a interação pessoal no processo (Almeida et al., 2009; David et al., 2015).

A perceção de competência varia consoante a idade das crianças, sendo que as crianças mais velhas têm mais capacidade de discriminar e avaliar as áreas em que são mais competentes, do que as crianças mais novas (Coelho et al., 2015; Tracey \& Ward, 1998).

Os estudantes mais velhos revelam mais dificuldade nas competências relacionadas com a gestão dos sentimentos, verifica-se que, na adolescência, é frequente a existência de altos níveis de stress e de estratégias decoping desadaptativas. Com o aumento da idade verifica-se um aumento das situações causadores de stress, sendo que os mais velhos experienciam mais situações de stress (incluindo situações com os pares, com a escola e com a família) e um aumento na complexidade das mesmas (Booker \& Dunsmore, 2016; Compas et al., 2017; Modecki et al., 2017; Zimmermann \& Iwanski, 2014). Estas múltiplas experiências negativas têm uma forte associação com expressões negativas de afeto entre as crianças mais velhas do que entre as mais novas (Zimmermann \& Iwanski, 2014). Assim, existem evidência na literatura que apontam para o facto de se verificar um aumento na utilização de estratégias de coping desadaptativas por parte 
dos jovens mais velhos (nomeadamente entre os 12 e os 15 anos) quando comparados com os mais novos, o que indica que a adolescência é caracterizada por variações ao nível da regulação emocional que influenciam a forma como os jovens lidam com as adversidades e desafios (Cracco et al., 2017). Segundo os profissionais, os alunos com PEI, apresentam menos competências socioemocionais do que os estudantes sem PEI em todas as dimensões da escala, com exceção à dimensão relacionada com a gestão de stress em que não foram encontradas diferenças estatisticamente significativas.

Os défices ao nível das competências sociais são comuns nos alunos com dificuldades de aprendizagem (Garner et al., 2014; Hen \& Goroshit, 2014; Nowicki et al., 2014). Na perspetiva dos profissionais, os alunos com dificuldades de aprendizagem são menos competentes ao nível académico, têm menos capacidade de interação social, exibem mais sinais de hiperatividade, falta de atenção e fraco ajustamento. Na perspetiva dos alunos, verificase um reportório deficitário ao nível da comunicação não verbal e da resolução de problemas (Kavale \& Forness, 1996; Kavale \& Mostert, 2004; Vaughn et al., 2001).

Bauminger e colaboradores (2005) concluem que crianças com dificuldades de aprendizagem apresentam maiores dificuldades ao nível do processamento da informação social e da capacidade de compreensão e expressão emocional. Bauminger e Kimhi-Kind (2008) acrescentam que crianças com dificuldades de aprendizagem apresentam maiores dificuldades ao nível do processamento da informação social, nomeadamente na capacidade de captar, representar mentalmente e interpretar as informações sociais, de clarificar objetivos, de procurar/gerar respostas sociais, na tomada de decisão sobre a melhor resposta após avaliar as consequências e possíveis resultados, e menor capacidade de regulação emocional.

Num estudo realizado por Wiener e Tardif (2004) concluise que as crianças com dificuldades de aprendizagem têm um baixo autoconceito académico, baixos níveis de competência social e apresentam problemas de comportamento, mais sentimentos de solidão e depressão, do que as crianças sem dificuldades de aprendizagem. Molina e Del Prette (2006) especificam que crianças com distúrbios ou dificuldades de aprendizagem apresentam características interpessoais que incluem, entre outros aspetos, tendência a serem mais agressivas, a apresentarem interações mais negativas com companheiros, a terem mais problemas de personalidade, menos comportamentos orientados para tarefa e a apresentarem um repertório menos elaborado de comportamentos interpessoais apropriados e desejáveis socialmente. As evidências na literatura apontam para o facto de que os alunos com dificuldades de aprendizagem experienciam mais sintomas de ansiedade (generalizada e social) e de depressão, constituindo um grupo com um risco mais elevado de desenvolver problemáticas ao nível socioemocional (Mammarella et al., 2014; Sahoo et al., 2015).

Segundo os profissionais, os estudantes alteraram o seu nível de competências socioemocionais após o programa de promoção de competências. Verifica-se o aumento de competências na maioria das dimensões da escala, nomeadamente ao nível das competências globais, nas competências avançadas, nas competências em lidar com sentimentos, competências alternativas à agressividade e competências de planeamento entre o momento pré e pós-intervenção.

Os programas de promoção de competências pessoais e sociais são considerados importantes formas de promover a saúde na escola, promovendo a escolha de um estilo de vida saudável e consequente melhor qualidade de vida, através do desenvolvimento das competências na criança e jovem para lidar consigo e com os outros, situações destress desafios proporcionados pelo ambiente (Gaspar et al., 2006). A promoção de competências sociais e pessoais deve ser de forma sistemática, continua e adequada às necessidades e faixa etária dos estudantes. A intervenção deve ser baseada em necessidades previamente identificadas junto dos diversos atores (estudantes, profissionais saúde e educação, pais, entre outros) e avaliadas. Deve ser realizada uma avaliação inicial, uma avaliação de processo, uma avaliação final e uma avaliação follow-up, que deve ser realizada pelo menos seis meses após o término da intervenção (Blum \& Dick, 2013; Gaspar, Cerqueira, Branquinho, \& Matos, 2018b; Matos et al., 2012, Reddy, 2013).

\section{CONCLUSÃO}

O presente estudo permite compreender e caracterizar a perceção dos professores e psicólogos de um centro educativo acerca das competências socioemocionais dos estudantes. São identificadas especificidades ligadas ao género, à idade e às necessidades educativas especiais. Segundo os profissionais, os mais novos, as meninas e os alunos sem necessidades educativas especiais, de modo geral, apresentam mais competências socioemocionais.

Segundo os profissionais, os estudantes alteraram o seu nível de competências socioemocionais após o programa de promoção de competências integradas. Verifica-se o aumento de competências na maioria das dimensões da escala.

Os resultados obtidos têm implicações para a investigação e intervenção. Sugere-se que para aumentar a eficácia das intervenções, os pais e os profissionais (educação, saúde, outros) devem possuir formação e competências adequadas e ajustadas às necessidades da população alvo. Os programas devem promover o bem-estar, as competências sociais e de resolução de problemas, as competências de autorregulação, a assiduidade e envolvimento/sucesso académico, ao invés de se focarem, apenas, na prevenção de comportamentos de risco.

As crianças mais velhas, meninas e meninos, por diferentes motivos, alunos carenciados, migrantes e alunos com necessidades educativas especiais, podem facilmente desistir de frequentar a escola devido ao facto de o currículo académico, os professores e os sistemas escolares como um todo, não 
serem capazes de colmatar as lacunas culturais, devido a uma compreensão limitada relativamente ao significado do conceito escola "para todos".

Para além disso, uma vez que os comportamentos de risco estão inseridos no contexto psicossocial, a intervenção preventiva deve ser implementada ao nível escolar, familiar e comunitário. Políticos, educadores, profissionais de saúde e outros grupos de profissionais, são encorajados a enfrentar o desafio de implementar intervenções efetivas, tendo por base a compreensão do conceito de género e de diversidade desenvolvimental.

\section{REFERÊNCIAS}

Almeida, G., Valentini, N. D. \& Berleze, A. (2009). Percepções de competência: Um estudo com crianças e adolescentes do ensino fundamental. Movimento, 15(1), 71-97.

Bandura, A. (1976). Social learning theory. New Jersey: Prentice-Hall.

Bauminger, N., Edelsztein, H. S., \& Morash, J. (2005). Social information processing and emotional understanding in children with LD. Journal of Learning Disabilities, 38(1), 45-61. doi:10.1177/0022219405038001040

Bauminger, N., \& Kimhi-Kind, I. (2008). Social information processing, security of attachment, and emotion regulation in children with learning disabilities. Journal of Learning Disabilities, 41(4), 315-332. doi:10.1177/0022219408316095

Blum, R. \& Dick, B. (2013). Strengthening global programs and policies for youth based on the emerging science. Journal of Adolescent Health 52(2), S1-S3. doi: https://doi.org/10.1016/j.jadohealth.2012.11.004

Booker, J. A., \& Dunsmore, J. C. (2017). Affective social competence in adolescence: Current findings and future directions. Social Development, 26(1), 3-20. doi: 10.111/sode.12193

Campbell, K., \& Peebles, R. (2014). Eating disorders in children and adolescents: state of the art review. Pediatrics, 134(3), 582-592. doi:10.1542/ peds.2014-0194

Chase, P. A., Hilliard, L. J., Geldhof, G. J., Warren, D. J., \& Lerner, R. M. (2014). Academic achievement in the high school years: The changing role of school engagement. Journal of Youth and Adolescence, 43(6), 884-896. doi:10.1007/s10964-013-0085-4

Choi, A. (2018). Emotional well-being of children and adolescents: Recent trends and relevant factors (OECD Education Working Papers, 169). Paris: OECD Publishing.

Coelho, V. A., Sousa, V., Marchante, M., \& Romão, A. M. (2015). Validação do questionário Autoconceito Forma 5 numa amostra de crianças e adolescentes portugueses. International Journal of Developmental and Educational Psychology, 1(1), 67-77.

Compas, B. E., Jaser, S. S., Bettis, A. H., Watson, K. H., Gruhn, M. A., Dunbar, J. P., ... \& Thigpen, J. C. (2017). Coping, emotion regulation, and psychopathology in childhood and adolescence: A meta-analysis and narrative review. Psychological Bulletin, 143(9), 939. doi:10.1037/ bul0000110

Cracco, E., Goossens, L., \& Braet, C. (2017). Emotion regulation across childhood and adolescence: Evidence for a maladaptive shift in adolescence. European Child \& Adolescent Psychiatry, 26(8), 909-921. doi:10.1007/ s00787-017-0952-8
David, R., Paixão, M. P., \& da Silva, J. T. (2015). Interesses e competências percebidas na infância: Um estudo com crianças do ensino básico. Revista Brasileira de Orientação Profissional, 16(1), 49-58.

Domitrovich, C. E., Durlak, J. A., Staley, K. C., \& Weissberg, R. P. (2017). Social-emotional competence: An essential factor for promoting positive adjustment and reducing risk in school children. Child Development, 88(2), 408-416. doi:10.1111/cdev.12739

Durlak, J. A., Weissberg, R. P., Dymnicki, A. B., Taylor, R. D., \& Schellinger, K. B. (2011). The impact of enhancing students' social and emotional learning: A meta-analysis of school-based universal interventions. Child Development, 82(1), 405-432. doi:10.1111/j.1467-8624.2010.01564.x

Eastabrook, J. M., Flynn, J. J., \& Hollenstein, T. (2014). Internalizing symptoms in female adolescents: Associations with emotional awareness and emotion regulation. Journal of Child and Family Studies, 23(3), 487-496. doi:10.1007/s10826-012-9705-y

Garner, P. W., Mahatmya, D., Brown, E. L., \& Vesely, C. K. (2014). Promoting desirable outcomes among culturally and ethnically diverse children in social emotional learning programs: A multilevel heuristic model. Educational Psychology Review, 26(1), 165-189. doi:10.1007/s10826-012-9705-y

Gaspar, T. (2010). Health-related quality of life in children and adolescents. Personal and social factors that promote quality of life. Riga: Lambert Academic Publishing.

Gaspar, T. \& Matos, M. (2015). Para mim é fácil - Escala de avaliação de competências pessoais e sociais. Psicologia, Saúde e Doenças, 16(2), $199-211$

Gaspar, T., Matos, M., Ribeiro, J., \& Leal, I. (2006). Qualidade de vida e bem-estar em crianças e adolescentes. Revista Brasileira de Terapias Cognitivas, 2(2), 47-60.

Gaspar, T., Matos, M. G., Ribeiro, J.L., Leal, I., Erhart, M., \& Ravens-Sieberer, U. (2012). Health-related quality of life in children and adolescents: Subjective well being. Spanish Journal of Psychology, 15(1), 177-186. http://dx.doi.org/10.5209/rev_SJOP.2012.v15.n1.37306

Gaspar, T., Rebelo, A., Mendonça, I., Albergaria, F., \& Matos, M. (2014). Subjective wellbeing and school failure in children and adolescents: Influence of psychosocial factors. International Journal of Development Research, 4(11), 2194-2199.

Gaspar, T., Matos, M.G., Pais Ribeiro, J., Leal, I., \& Albergaria, F. (2014). Psychosocial Factors Related to Bullying and Victimization in Children and Adolescents. Health Behavior \& Policy Review, 1(6), 452-459. doi: http:// dx.doi.org/10.14485/HBPR.1.6.3

Gaspar, T., \& Balancho, L. (2016). Factores pessoais e sociais que influenciam o bem-estar subjectivo: diferenças ligadas estatuto socioeconómico. Ciência e Saúde Coletiva, 22(4), 1373-1380.

Gaspar, T., Bilimória, H., Albergaria, F., Matos, M.G. (2016). Children with special education needs and subjective well-being: Social and personal influence. International Journal of Disability, Development and Education, 63(5), 500-513.

Gaspar, T., Matos, M., Luszczynska, A. \& De Wit, J. (2016). Socio-economic and self-regulatory influences on eating behavior in children and adolescents from four European countries. North American Journal of Psychology, 18(1), 177-192.

Gaspar, T., Cerqueira, A., Branquinho, C. \& Matos, M. G. (2018a). The effect of a social-emotional school-based intervention upon social and personal skills in children and adolescents. Journal of Education and Learning, 7(6), 57-66. doi:10.5539/jel.v7n6p57 
Gaspar, T., Cerqueira, A., Branquinho, C. \& Matos, M. (2018b). Dimensions of social and personal skills in children and adolescents: Age and gender differences. International Journal of Development Research, 8(1), 18394-18400.

Goldman, E., Stamler, J., Kleinman, K., Kerner, S. \& Lewis, O. (2016). Child mental health: Recent developments with respect to risk, resilience and interventions. In M. R. Korin (Ed.), Health promotion for children and adolescents (pp. 99-124). New York: Springer.

Goldstein, A. P. \& McGinnis, E. (1997). Skillstreaming the adolescents: New strategies and perspectives for teaching prosocial skills (rev. ed.). Champaign: Research Press.

Hampel, P., \& Petermann, F. (2005). Age and gender effects on coping in children and adolescents. Journal of Youth and Adolescence, 34(2), 73-83. doi:10.1007/s10964-005-3207-9

Hampel, P., \& Petermann, F. (2006). Perceived stress, coping, and adjustment in adolescents. Journal of Adolescent Health, 38(4), 409-415. doi:10.1016/j.jadohealth.2005.02.014

Harter, S. (1978). Effectance motivation reconsidered. Human Development, 21(1), 34-64. doi:10.1159/000271574

Hen, M., \& Goroshit, M. (2014). Academic procrastination, emotional intelligence, academic self-efficacy, and GPA: A comparison between students with and without learning disabilities. Journal of Learning Disabilities, 47(2), 116-124. doi:10.1177/0022219412439325

Kavale, K. A. \& Forness, S. R. (1996). Social skill deficits and learning disabilities: A meta-analysis. Journal of Learning Disabilities, 29(3), 226-237. doi:10.1177/002221949602900301

Kavale, K. A., \& Mostert, M. P. (2004). Social skills interventions for individuals with learning disabilities. Learning Disability Quarterly, 27(1), 31-43. doi:10.2307/1593630

Mammarella, I. C., Ghisi, M., Bomba, M., Bottesi, G., Caviola, S., Broggi, F., \& Nacinovich, R. (2014). Anxiety and depression in children with nonverbal learning disabilities, reading disabilities, or typical development. Journal of learning disabilities, 49(2), 130-139. doi:10.1177/0022219414529336

Matos, M. G. (2005). Comunicação e gestão de conflitos e saúde na escola. Lisboa: FMH.

Matos, M. G. (2008). Adolescência, psicologia da saúde e saúde pública. In M. G. Matos (Ed.). Comunicação, gestão de conflitos e saúde na escola (pp. 10-31). Lisboa: CDI/FMH.

Matos, M. G., \& Sampaio, D. (2009). Jovens com saúde: Diálogos com uma geração. Lisboa: Texto.

Matos, M. G., Simões, C., Gaspar, T., \& Equipa do Projecto Aventura Social (2009). Violência entre pares no contexto escolar em Portugal, nos últimos 10 anos. Interaç̧ões, 13, 98-124.

Matos, M.G., \& Tomé, G. (Org.). (2012). Aventura social: Promoção de competências e do capital social para o empreendedorismo com saúde na escola e na comunidade. Lisboa: Placebo.

Matos, M. G., Gaspar, T., Ferreira, M., Tomé, G., Camacho, I., Reis, M., Melo, P., Simões, C., Machado, R., Ramiro, L., \& Equipa Aventura Social (2012). Keeping a focus on self-regulation and competence: "find your own style", A school-based program targeting at risk adolescents. Journal of Cognitive and Behavioral Psychotherapies, 12(1), 39-48.

Matos, M.G., Tomé, G., Gaspar, T., Cicognani, E. \& Moreno, M.C. (2016). Youth mental health in Portugal, Italy and Spain: Key challenges for improving well-being. The European Health Psychologist, 18(3), 128-133.
McConnell, M. M., Memetovic, J., \& Richardson, C. G. (2014). Coping style and substance use intention and behaviour patterns in a cohort of BC adolescents. Addictive Behaviors, 39(10),1394-1397. doi:10.1016/j. addbeh.2014.05.018

Modecki, K. L., Zimmer-Gembeck, M. J., \& Guerra, N. (2017). Emotion regulation, coping, and decision making: Three linked skills for preventing externalizing problems in adolescence. Child Development, 88(2), 417426. doi:10.1111/cdev.12734

Moksnes, U. K., Espnes, G. A., \& Haugan, G. (2014). Stress, sense of coherence and emotional symptoms in adolescents. Psychology \& Health, 29(1), 32-49. doi:10.1080/08870446.2013.822868

Molina, R. C. M., \& Del Prette, Z. A. P. (2006). Funcionalidade da relação entre habilidades sociais e dificuldades de aprendizagem. Psico-USF, 11(1), 53-63. doi:10.1590/S1413-82712006000100007

Morris, J. L., \& Rushwan, H. (2015). Adolescent sexual and reproductive health: The global challenges. International Journal of Gynecology \& Obstetrics, 131 (Suppl 1), S40-S42. doi:10.1016/j.ijgo.2015.02.006

Nowicki, E. A., Brown, J., \& Stepien, M. (2014). Children's thoughts on the social exclusion of peers with intellectual or learning disabilities. Journa of Intellectual Disability Research, 58(4), 346-357. doi: 10.1111/jir.12019

Reddy, K. (2013). Evidence-based interventions in low- and middle-income countries: The tigress awakens. Journal of Adolescent Health, 52(2, Suppl 2), S5-S6. doi: http://dx.doi.org/10.1016/j.jadohealth.2012.11.006

Riediger, M., \& Klipker, K. (2014). Emotion regulation in adolescence. In J. J. Gross (Ed.), Handbook of emotion regulation (pp. 187-202). New York: Guilford.

Sahoo, M. K., Biswas, H., \& Padhy, S. K. (2015). Psychological co-morbidity in children with specific learning disorders. Journal of Family Medicine and Primary Care, 4(1), 21-25. doi:10.4103/2249-4863.152243

Sancassiani, F., Pintus, E., Holte, A., Paulus, P., Moro, M. F., Cossu, G., ... Lindert, J. (2015). Enhancing the emotional and social skills of the youth to promote their wellbeing and positive development: a systematic review of universal school-based randomized controlled trials. Clinical practice and epidemiology in mental health, 11 (Suppl $1 \mathrm{M} 2$ ), 21-40. doi: http:// doi.org/10.2174/1745017901511010021

Spear, L. P. (2015). Adolescent alcohol exposure: Are there separable vulnerable periods within adolescence?. Physiology \& behavior, 148 122-130. doi:10.1016/j.physbeh.2015.01.027

Taylor, R. D., Oberle, E., Durlak, J. A., \& Weissberg, R. P. (2017). Promoting positive youth development through school-based social and emotional learning interventions: A meta-analysis of follow-up effects. Child development, 88(4), 1156-1171. doi:10.1111/cdev.12864

Tracey, T. G., \& Ward, C. C. (1998). The structure of children's interests and competencies perceptions. Journal of Counselling Psychology, 45(3), 290-303. doi:10.1037/0022-0167.45.3.290

Ungar, M., Russell, P., \& Connelly, G. (2014). School-based interventions to enhance the resilience of students. Journal of Educational and Developmental Psychology, 4(1), 66. doi:10.5539/jedp.v4n1p66

Vaughn, S., \& Elbaum, B., \& Boardman, A. G. (2001). The social functioning of students with learning disabilities: implications for inclusion. Exceptionality, 9(1/2), 47-65. doi:10.1080/09362835.2001.9666991

Weissberg, R. P., Durlak, J. A., Domitrovich, C. E., \& Gullotta, T. P. (Eds.) (2015). Social and emotional learning: Past, present, and future. In J. A Durlak, C. E. Domitrovich, R. P. Weissberg, \& T. P. Gullotta (Eds.), Handbook of social and emotional learning: Research and practice (pp. 3-19). New York: Guilford. 
Wiener, J., \& Tardif, C. Y. (2004). Social and emotional functioning of children with learning disabilities: Does special education placement makes a difference? Learning Disabilities Research and Practice, 19(1), 20-32. doi:10.1111/j.1540-5826.2004.00086.x

World Health Organization (WHO). (2014). Health for the world's adolescents: A second chance in the second decade. Geneva: World Health Organization.
Zimmermann, P., \& Iwanski, A. (2014). Emotion regulation from early adolescence to emerging adulthood and middle adulthood: Age differences, gender differences, and emotion-specific developmental variations. International Journal of Behavioral Development, 38(2), 182-194. doi:10.1177/0165025413515405 